\title{
The long non-coding RNA PCGEM1 is regulated by androgen receptor activity in vivo
}

\author{
Abhijit Parolia ${ }^{1,2,3}$, Francesco Crea ${ }^{2,3,5}$, Hui Xue ${ }^{2,3}$, Yuwei Wang ${ }^{2,3}$, Fan Mo ${ }^{3}$, Varune Rohan Ramnarine ${ }^{3}$, \\ Hui Hsuan Liu'2, Dong Lin 2,3,5, Nur Ridzwan Nur Saidy ${ }^{1,2}$, Pier-Luc Clermont 2,4, Hongwei Cheng 2,3, Colin Collins ${ }^{3}$, \\ Yuzhuo Wang ${ }^{2,3^{*}+}$ and Cheryl D Helgason ${ }^{2,6^{*}+}$
}

\begin{abstract}
Background: Long non-coding RNAs (IncRNAs) can orchestrate oncogenic or tumor-suppressive functions in cancer biology. Accordingly, PCGEM1 and PRNCR1 were implicated in progression of prostate cancer (PCa) as transcriptional co-regulators of the androgen receptor (AR). However, these findings were recently refuted asserting that neither gene physically binds to the AR. Despite evidence for differing AR transcriptional programs in vivo and in vitro, studies investigating AR-regulation of these genes hitherto have only been conducted in vitro. Here, we further examine the relevance of PCGEM1 and PRNCR1 in PCa, and their relationship with AR signaling, using patient-derived xenograft models.
\end{abstract}

Findings: RNA sequencing of two distinct androgen-dependent models shows PCGEM1 to be considerably expressed, while PRNCR1 showed scant basal expression. PCGEM1 was sharply down-regulated following castration and up-regulated upon AR activation in vivo. However, we found no parallel evidence following AR stimulation in vitro. A PCGEM1-associated gene expression signature (PES) was significantly repressed in response to androgen ablation therapy and in hormone-refractory versus hormone-naïve PCa patients. Furthermore, we found PCGEM1 was uniformly distributed in PCa cell nucleus and cytoplasm which remained unaltered upon AR transcriptional activation. PCGEM1 was up-regulated in primary PCa but not in metastasized PCa. Accordingly, the PES was significantly down-regulated in advanced and higher grade PCa patients from multiple independent studies.

Conclusion: Our results demonstrate PCGEM1 as an in vivo androgen-regulated transcript with potential nuclear and/or cytoplasmic function(s). Importantly, the clinical expression profile of PCGEM1 implicates it in the early stages of PCa warranting further research in this direction.

Keywords: Long non-coding RNAs, InCRNAs, PCGEM1, PRNCR1, PCAT18, Androgen receptor, AR regulation, Sub-cellular localization, Prostate cancer

\section{Introduction}

In recent years, long non-coding RNAs (lncRNAs) have emerged as major contributors to cellular homeostasis as well as initiation and progression of numerous diseases [1], including prostate cancer (PCa) [2]. The latest GENCODE v7 project annotated 14,880 human lncRNA transcripts with only a few characterized to date [3]. Of the lncRNAs functionally validated in various human malignancies, a majority have been identified as constituents

\footnotetext{
* Correspondence: ywang@bccrc.ca; chelgaso@bccrc.ca

'Equal contributors

${ }^{2}$ Experimental Therapeutics, BC Cancer Research Centre, 675 West 10th

Avenue, Vancouver, BC V5Z 1L3, Canada

Full list of author information is available at the end of the article
}

of oncogenic or tumor suppressive pathways [4,5]. Some prominent lncRNAs implicated in prostate carcinogenesis and its progression include prostate cancer associated transcript 1 (PCAT1) [6], second chromosome locus associated with prostate 1 (SChLAP1) [7], prostate cancer associated 3 $(P C A 3)$ [8], prostate cancer gene expression marker 1 (PCGEM1; aka PCAT9) [9] and prostate cancer associated non-coding RNA 1 (PRNCR1; aka PCAT8) [10]. Notably, SChLAP1 has been extensively validated in the clinics as a biomarker of aggressive PCa [11] and PCA3 is currently used in diagnostic tests [12]. Recently, we described PCAT18 (aka Loc728606, Linc01092) as a mediator of metastatic progression based on expression profiling of our 
patient-derived PCa xenograft models from the Living Tumor Laboratory (LTL) [13]. PCGEM1, a highly prostate-specific transcript, was one of the first oncogenic lncRNAs to be described in PCa [9]. Subsequently, its over-expression was reported to attenuate the apoptotic response [14] and also promote cell proliferation and colony formation [15]. On the other hand, PRNCR1 is not as well investigated, although its knockdown reportedly inhibits cell viability [10]. Recently, both of these lncRNAs occupied center stage due to their labeling as androgen receptor (AR)-interacting genes [16] - a claim now disputed [17].

$\mathrm{AR}$ is a ligand-responsive regulatory protein that mediates the effector functions of androgenic hormones in PCa. It is well established that sustained AR activity is indispensable for PCa cell survival and disease progression, even following androgen-deprivation therapy [18-20]. This "AR addiction" has led to many studies investigating genes serving as conduits for aberrant restoration of AR activity in recurrent tumors as potential therapeutic targets. In this regard, Srikantan et al. described PCGEM1 as an AR regulated gene. Later on, PCGEM1 acting in complicity with PRNCR1 was shown to physically bind to the AR, thereby facilitating its ligand-independent transcriptional activity in castration resistant PCa (CRPC) [16]. In contrast, a recent publication indicated that neither PCGEM1 nor PRNCR1 interacted with the AR to render androgen-independence, and that both genes had no prognostic relevance in PCa [17]. Furthermore, the latter study found no evidence of PCGEM1 and PRNCR1 transcripts being AR regulated.

Notably, all published data on the relationship between $\mathrm{AR}$ and PCGEM1/PRNCR1 hitherto have been derived from in vitro experiments, using androgen-sensitive LNCaP cells $[9,17]$. There is substantial evidence that transcriptional regulation of genes by a transcription factor is highly dynamic and cellular context-specific [21]. In this light, AR was recently demonstrated to induce varied and distinct $A R$ transcriptional programs in patient tumor tissue as opposed to PCa cell lines [22]. This puts in doubt the suitability of cell line models for studying the transcriptional activity of the AR. In view of this, we set out to specifically investigate whether PCGEM1 and PRNCR1 are relevant in $\mathrm{PCa}$ and/or regulated by AR using our LTL patient-derived xenograft PCa models. The LTL has established a large panel of patient-derived PCa xenograft models that, unlike cell lines, retain key biological properties of the original malignancy, including histopathology, genomic profile, cellular heterogeneity, and invasive and metastatic ability [23].

\section{Findings and discussion}

As a first step, we profiled our two AR+/androgen dependent PCa xenograft models - LTL-331 and LTL-313B - for expression of both lncRNAs using RNA
Sequencing. While PCGEM1 was considerably expressed ( $>500$ FPKM; fragments per kilobase of exon per million fragments mapped), the expression of PRNCR1 was $<8$ FPKM in both models (Additional file 1: Table S1). Such scant expression of PRNCR1 raises serious questions about its biological relevance in AR-dependent PCa. Notably, this negligible expression of PRNCR1 is in accordance with a more extensive clinical dataset that was recently published [17]. Together, these data weaken the claim made by Yang et al. [16] that PRNCR1 plays a vital role in directing the transcriptional activity of AR. We, therefore, focused solely on PCGEM1 for the remainder of our study.

\section{AR regulates expression of PCGEM1 in vivo}

We performed surgical castration (androgen ablation) of mice bearing the LTL-331 PCa xenograft. Tissue samples were collected just prior to castration and 3 weeks after castration, and were analyzed for gene expression. We observed a significant $>500$-fold decrease in PCGEM1 expression post-castration relative to the pre-castration level (Figure 1A). Notably, this down-regulation was persistent (>180-fold) even twelve weeks following castration (Additional file 2: Figure S1). In agreement with this data, qPCR analysis on RNA collected before and 12 weeks after castration from a second in vivo model, LTL-313B, confirmed the down-regulation of PCGEM1 (>70-fold; Figure 1B). In both models, reduced AR activity in response to castration was confirmed by the comparable down-regulation of PCAT18 (Figure 1A,B), an AR-regulated lncRNA [13], and reduction in serum prostate-specific antigen (PSA) levels (at least $>45$-fold; Figure $1 C$ ). These findings led us to investigate the response of PCGEM1 to AR stimulation in vivo. This was achieved by using intact mouse hosts supplemented with or without pure testosterone at two distinct dosages $(1.0 \mathrm{mg}$ and $5.0 \mathrm{mg} / \mathrm{mouse}$ ) for PCa xenotransplantation of the LTL-331 tumor line. Corresponding to the dosages, serum testosterone levels of $3.96 \pm 0.679 \mathrm{ng} / \mathrm{ml}$ and $20.6 \pm 14.1 \mathrm{ng} / \mathrm{ml}$ were achieved in the testosterone supplemented animals versus $1.95 \pm 0.106 \mathrm{ng} / \mathrm{ml}$ in the intact hosts. Notably, the hosts supplemented with pure testosterone showed a dose-dependent up-regulation of PCGEM1, from 5-fold to greater than 22-fold, relative to the hosts without the augmented testosterone (Figure 1D). This coincided with a comparable PCAT18 up-regulation and serum PSA jump of $>2$-fold at both dosages.

Notably, previous in vitro studies investigating ARregulation of PCGEM1 produced conflicting results. Srikantan et al. [9] concluded PCGEM1 is AR-regulated, while Prensner et al. [17] found no up-regulation of PCGEM1 upon AR stimulation. The reason for this discrepancy could lie in the varied experimental conditions employed; in particular, the use of different agonists 


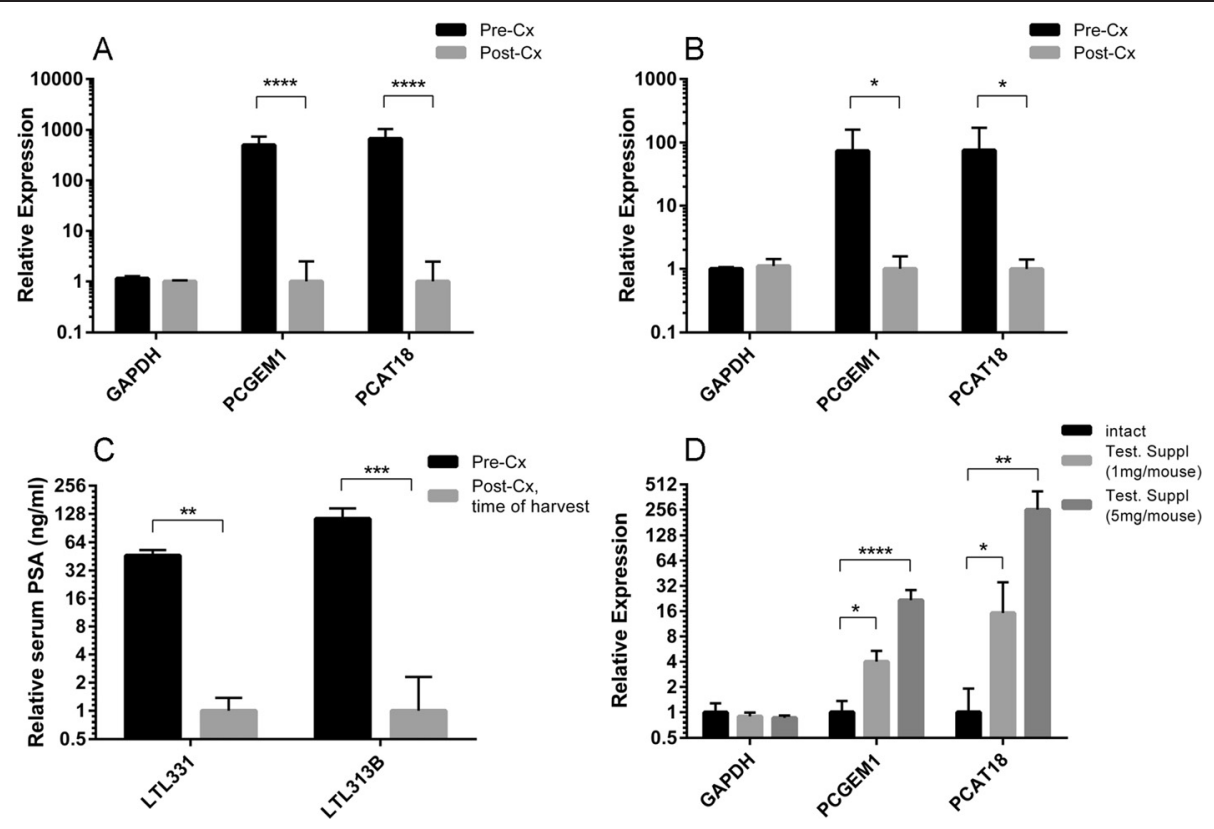

Figure 1 Expression of PCGEM1 in response to AR manipulation in vivo. A, Expression (qPCR) of the labeled genes in LTL-331 tumor line from intact mice supplemented with testosterone (Test.) $(n=3 ; 5.0 \mathrm{mg} /$ mouse) and 3 weeks after surgical castration $(n=4)$. Expression of all genes is referenced to average expression levels of HPRT1 and GAPDH and is expressed relative to the gene's expression under castrate conditions. ${ }^{* * * *} \mathrm{p}<0.0001$ (unpaired, 2-tailed $T$ test). B, Expression of the labeled genes (qPCR) in LTL-313B tumor line pre-castration ( $\left.n=3\right)$ and 12 weeks after castration $(n=3)$. Expression data is referenced and represented as described in the legend of Figure $1 A .{ }^{*} p<0.05$ (unpaired, 2 -tailed $T$ test). C, Serum PSA levels in corresponding animals bearing LTL-331 or LTL-313B xenograft in A and B, respectively, just prior to castration and at the time of tissue collection after castration. Data is depicted relative to serum PSA values at post-castration time-points. ${ }^{* *} p<0.01,{ }^{* * *} p<0.001$ (unpaired, 2-tailed $T$ test) $\mathbf{D}$, Expression levels ( $\mathrm{PPCR}$ ) of the labeled genes in intact mouse hosts $(n=3)$ or intact hosts supplemented with two distinct dosages of pure testosterone (Test.) $(1.0 \mathrm{mg} / \mathrm{mouse}$ or $5.0 \mathrm{mg} / \mathrm{mouse}, \mathrm{n}=4$ and $\mathrm{n}=3$ respectively). Expression data is referenced as in Figure $1 \mathrm{~A}$ and is depicted relative to the gene's expression in intact mice. ${ }^{*} p<0.05,{ }^{* *} p<0.01,{ }^{* * * *} p<0.0001$ (2-Way ANOVA and Tukey's post-test), For all the sub-figures, columns represent mean expression value for the biological replicates (each gene is quantified in triplicates), and bars represent standard deviation. TaqMan assay IDs for all the genes are listed in "Additional file 1: Table S4".

(viz. dihydrotestosterone (DHT) and synthetic R1881) for AR activation. Although the patterns of DHT-mediated and R1881-mediated gene expression changes are largely parallel, they do not completely overlap [24]. Consequently, we repeated this experiment using DHT, a physiological AR ligand, coupled with the sensitive TaqMan qPCR for gene quantification. Two $\mathrm{AR}+\mathrm{PCa}$ cell line models that expressed the highest levels of PCGEM1, LNCaP and VCaP, were chosen for the in vitro studies (Additional file 2: Figure S2). DHT treatment (at $6 \mathrm{~h}$ and $12 \mathrm{~h}$ ) induced a modest (1.8 - 2.2-fold) up-regulation of PCGEM1 in LNCaP cells (Figure 2A). However, the activation of PCGEM1 did not continue to escalate in a manner similar to that of canonical AR-regulated genes such as PSA and PCAT18 over extended time-points (12 h and $24 \mathrm{~h})$. Similar observations were made in $\mathrm{VCaP}$ cells upon treatment with DHT (10nM; Figure 2B). Most remarkably, the weak AR-activation of PCGEM1 in vitro is in accordance with the recent observation that genes actively regulated by the $\mathrm{AR}$ in patient tumor tissue show a strong in vivo response to castration but a distinctly lesser response to androgen stimulation in vitro [22]. Notably, also contradicting the original findings [9], no significant increase in PCGEM1 expression was observed in vitro (at $6 \mathrm{~h}, 12 \mathrm{~h}$ or $24 \mathrm{~h}$ ) following stimulation of LNCaP cells with R1881 - a synthetic AR ligand (at 10nM; Figure 2C). Similar results were obtained on treatment with a super-physiological concentration of DHT (100nM; Figure 2D). Taken together, our results categorically demonstrate that substantial regulation of PCGEM1 expression by androgen occurs exclusively in vivo.

\section{AR activation does not alter the uniform sub-cellular localization of PCGEM1}

It was previously suggested that a direct AR-PCGEM1 interaction regulates AR's transcriptional activity [16]. In line with this mechanistic model, PCGEM1's suspected function as a transcriptional co-regulator would imply it to be predominantly contained in the nucleus, in particular when the AR is transcriptionally active. However, sub-cellular localization of PCGEM1 had never been investigated. Addressing this issue, we performed cellular fractionation of $\mathrm{PCa}$ patient-derived xenograft cells to 

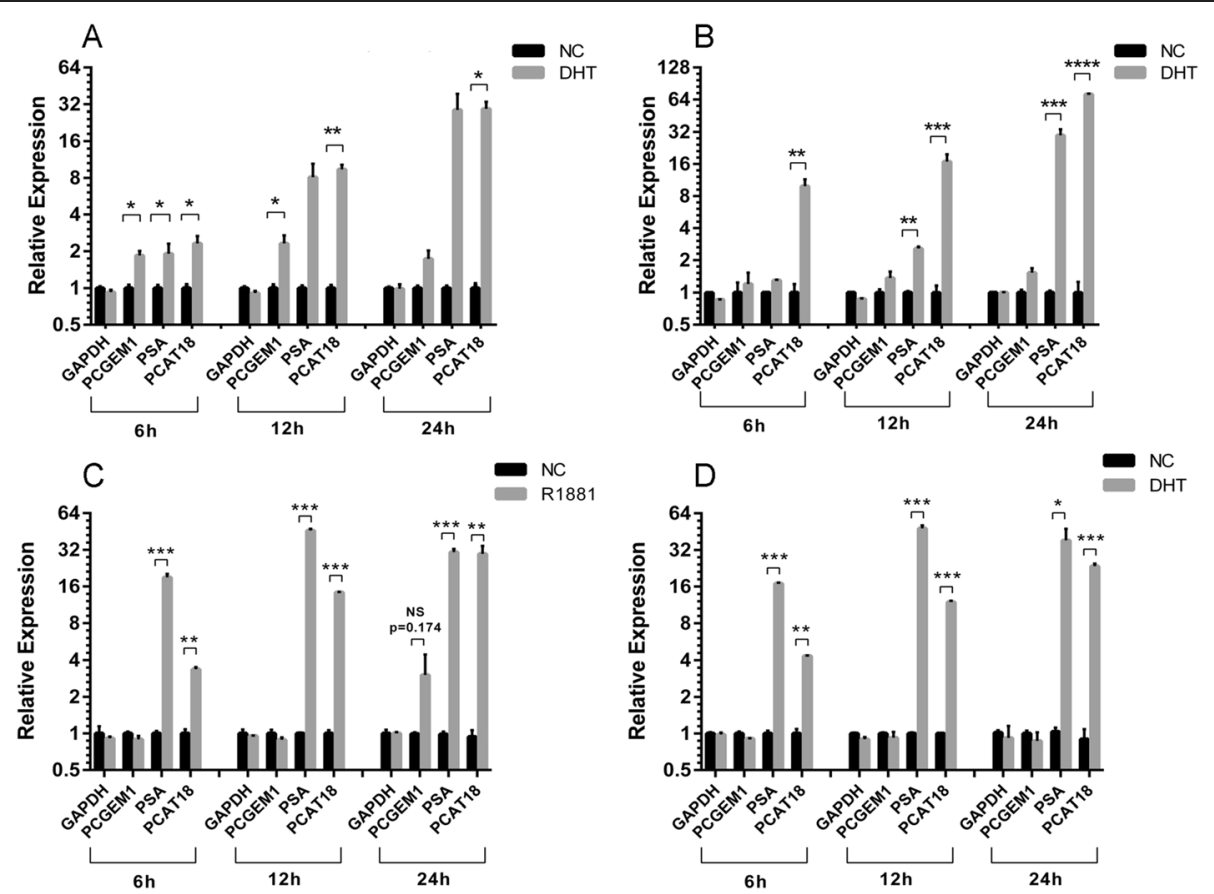

Figure 2 Expression of PCGEM1 in response to AR activation in vitro. Expression levels (qPCR) of the labeled genes in $\mathbf{A}, L N C a P$ cells and $\mathbf{B}$, VCaP cells treated with DHT at 10nM for $6 \mathrm{~h}, 12 \mathrm{~h}$ and $24 \mathrm{~h}$. C, Expression (qPCR) of the labeled genes in LNCaP cells at $6 \mathrm{~h}, 12 \mathrm{~h}$ and $24 \mathrm{~h}$ after treatment with R1881 (10nM) or D, with a super-physiological dosage of DHT (100nM). For all the sub-figures, the columns represent mean expression value ( 2 independent experiments with each gene quantified in triplicates), bars represent standard deviation. NS, not significant; ${ }^{*} p<0.05 ;{ }^{* *} p<0.01 ;{ }^{* *} p<0.001 ;{ }^{* * *} p<0.0001$ (unpaired, 2-tailed $T$ test).

isolate the nuclear and cytoplasmic RNA fractions, and quantified gene expression.

Our data shows that PCGEM1 is evenly distributed between the nucleus and the cytoplasm of LTL-331 cells harvested from intact mouse hosts or intact hosts supplemented with testosterone (Figure 3A,B). Expected cytoplasmic localization of GAPDH and Actin (>85\% for both) and nuclear localization of small nucleolar RNA 55 (snoRNA55) and MALAT1 (>80\% for both) authenticate our findings. A similar sub-cellular distribution of PCGEM1 was observed in DHT treated and untreated LNCaP cells as well (Additional file 2: Figure S3A, B). While these results do not conclusively rule out the possibility that PCGEM1 may be interacting with the AR, they demonstrate for the first time that PCGEM1 is not selectively localized in the nucleus; or is shuttled to it upon transcriptional activation of the AR. These findings also highlight the gap in our understanding of lncRNAs with proposed
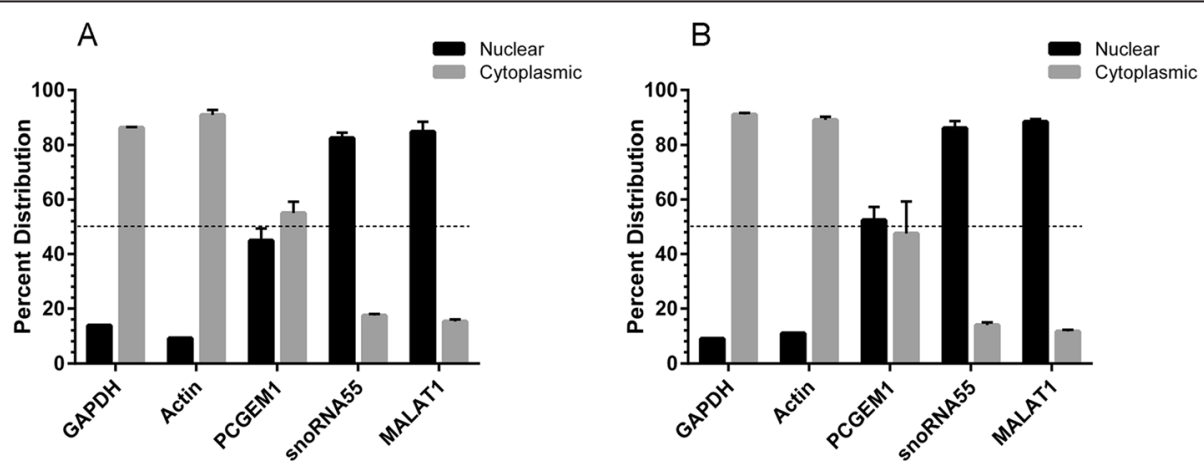

Figure 3 Subcellular localization of PCGEM1 with or without AR activation in vivo. Sub-cellular localization of PCGEM1, GAPDH mRNA, Actin mRNA, snoRNA55 and MALAT1 in $\mathbf{A}$, LTL-331 cells harvested from intact mouse hosts $(n=2)$ and $\mathbf{B}$, intact hosts supplemented with pure testosterone (Test. Suppl; $5.0 \mathrm{mg} /$ mouse, $\mathrm{n}=2$ ). For each gene, expression in nuclear and cytoplasmic fractions is represented as a percentage of the total expression. The dashed line indicates the 50\% distribution mark. The columns represent mean \% distribution value for the biological replicates (each gene is quantified in triplicates), and the bars represent standard deviation. 
cell compartment-specific functions. The unresolved question is whether IncRNAs with nuclear roles are contained in the nucleus throughout their lifetime, or do they also shuttle to the cytoplasm where they can adopt additional roles? For instance, H19, one of the most well characterized lncRNAs, is associated with both nuclear and cytoplasmic functions i.e. chromatin modulation [25] and micro-RNA generation [26,27], respectively. Collectively, our results warrant further verification of the alleged function of PCGEM1 as a transcriptional co-regulator of the AR, in addition to investigating its potential role in the cytoplasm. For detailed information about all the experiments refer to "Additional file 3: Supplementary methods and materials."

\section{PCGEM1 is implicated in early stages of PCa}

Our results to this point indicate PCGEM1 to be an in vivo androgen regulated gene, with uniform distribution in $\mathrm{PCa}$ cell nucleus and cytoplasm. One remaining question, however, is the stage of $\mathrm{PCa}$ in which PCGEM1 might be biologically most relevant. To this end we investigated a patient database consisting of microarray and clinical data on 131 primary $\mathrm{PCa}, 19$ metastasized $\mathrm{PCa}$ and 29 normal prostatic tissue samples [28]. Here we observed that PCGEM1 was significantly up-regulated only in primary PCa but not in metastasized PCa relative to normal prostate tissue. In fact, PCGEM1 expression was significantly down-regulated in metastasized tumors relative to primary tumors (Additional file 2: Figure S4). Adding to a recent study discrediting the involvement of PCGEM1 in progression of localized PCa to CRPC [17], our analysis further raises the question of the biological relevance of PCGEM1 in survival and proliferation of metastasized PCa cells themselves. In view of this, PCGEM1 is most likely implicated only in the early stages of the disease warranting further experimental validation. To explore this further, we performed Pearson's Correlation analysis to identify genes that are positively associated with PCGEM1 expression, hereafter referred to as PCGEM1-associated gene expression signature (PES) (Additional file 1: Table S2), and uploaded the list into the Oncomine database. As previously demonstrated for PCGEM1, the PES was also significantly up-regulated in $\mathrm{PCa}$ relative to other neoplasms and in $\mathrm{PCa}$ versus normal prostate tissue (Table 1). Corroborating the relevance of PCGEM1 in early stages of $\mathrm{PCa}, \mathrm{PES}$ was consistently repressed in metastatic and high Gleason $\mathrm{PCa}$ relative to primary and low Gleason neoplasms, respectively (Table 1). Notably, this trend was observed in several independent patient cohorts totaling more than 1100 samples. Accordingly, the PES was significantly repressed in patients exhibiting poor clinical outcomes.

A review of literature also reveals that PCGEM1 is upregulated in normal prostate epithelial cells of men with a family history of $\mathrm{PCa}$ [15] and certain single-nucleotide polymorphisms in the PCGEM1 gene are associated with a higher risk of PCa development in Chinese men [29]. Together, these findings support the testable hypothesis that PCGEM1 is more important in the early stages, possibly initiation, of primary $\mathrm{PCa}$. Interestingly, the PCGEM1 signature was also under-expressed in hormone-refractory $\mathrm{PCa}$ relative to hormone-naïve PCa (Table 1); and the pathway analysis revealed PES to be most significantly down-regulated $(\mathrm{p}=5.47 \mathrm{E}-14$, odds ratio $=121.4)$ in patients receiving androgen-deprivation therapy (Additional file 1: Table S3) lending further support to in vivo AR-regulation of PCGEM1.

Our work brings in light an even more pertinent concern with exploring AR transcriptional activity using cell line models. Recently, cell line models in accordance with their metastatic origin were demonstrated to exhibit the AR regulatory program that is active in metastatic advanced PCa as opposed to primary PCa [22]. The AR regulatory activity in cultured cell line models (including LNCaP) had a greater overlap to CRPC (31\% overlap) than untreated primary $\mathrm{PCa}$ (merely $3 \%$ overlap) (see [22]). Besides the absent cell microenvironment, this

Table 1 PCGEM1-associated expression signature in PCa patient samples

\begin{tabular}{lllll}
\hline Concept & Studies (Up/Down) & P-value & Odds ratio & Total samples \\
\hline PCa vs. Other Neoplasms & $1 / 0$ & $4.44 \mathrm{E}-15$ & 30.9 & 1468 \\
PCa vs. Normal Prostate & $5 / 0$ & $1.88 \mathrm{E}-10-2.00 \mathrm{E}-03$ & $14.5-4.5$ & 299 \\
Metastatic PCa vs. Primary PCa & $0 / 6$ & $6.69 \mathrm{E}-13-5.00 \mathrm{E}-03$ & $27.0-4.5$ & 448 \\
High Gleason PCa vs. Low Gleason PCa & $0 / 5$ & $8.01 \mathrm{E}-11-6.00 \mathrm{E}-3$ & $16.8-5.0$ & 676 \\
Hormone-Refractory PCa vs. Hormone-naïve PCa & $0 / 1$ & $4.74 \mathrm{E}-05$ & 9.6 & 20 \\
Clinical Outcomes & & & $8.4-5.1$ \\
$\quad$ Reoccurrence at 3 or 5 years & $0 / 2$ & $1.45 \mathrm{E}-06-4.52 \mathrm{E}-04$ & 168 \\
$\quad$ Dead at 3 or 5 years & $0 / 2$ & $4.65 \mathrm{E}-07-9.02 \mathrm{E}-04$ & $20.6-7.8$ & 721 \\
\hline
\end{tabular}

The MSKCC database was queried using cBioPortal for genes that are co-expressed with PCGEM1 (Pearson's Correlation > 0.50), which were then uploaded into the Oncomine database (thresholds: $p$-value $<0.01$, odds ratio $>2$ ). Only clinically-related Oncomine concepts were analyzed and are listed in the first column. Here, PCGEM1 expression in PCa was compared to 15 other neoplasms. The second column summarizes the total number of independent studies showing either up- or down-regulation of PES for the corresponding concept. "Total Samples" reflect the grand total of number of patients in every study that met the threshold for the corresponding concept. Oncomine (Compendia Bioscience) was used for statistical analysis. 
describes further limitations of cell line models for investigating AR-regulation, in particular for genes with a clinical expression profile akin to that of PCGEM1. Simultaneously, it underscores the importance of using clinically relevant models that best represent the original malignancy. With mounting evidence for lncRNAs implicated in carcinogenesis and cancer progression, we regard lncRNAs as essential in decrypting cancer cell biology. Our study corroborates the irrelevance of PRNCR1 in $\mathrm{PCa}$, and confirms PCGEM1 as an in vivo AR-regulated transcript as well as rationalizes its oncogenic involvement in early stages of the disease.

\section{Additional files}

Additional file 1: Table S1. RNA-Sequencing data for PCGEM1 and PRNCR1 in LTL xenograft models. Transcript name, ensembl ID, transcript class, chromosomal coordinates and FPKM values in LTL-331 and LTL-313B for GAPDH (control), PCGEM1 and PRNCR1. Table S2. PCGEM1-associated expression signature (PES) in PCa Samples. PCGEM1 was queried using CBioPortal in the prostate adenocarcinoma (MSKCC, Cancer Cell 2010) cancer study for mRNA expression data. List of genes with the highest expression correlation with PCGEM1 (pearson's correlation >0.50) was obtained from the "Co-Expression" module on cBioPortal. A total of 29 genes, listed in the first column, were significantly co-expressed with PCGEM1. Second and third columns list the Pearson's and Spearman's correlation values that were obtained from the cBioPortal analysis. Table S3. Literature-defined concepts associated with PES. All transcripts that positively associated with PCGEM1 (see Additional file 1: Table S2) were uploaded into the Oncomine database and analyzed for "literature-defined concepts" (thresholds: P-value $<0.01$. odds ratio $>2$ ). Here, we show only the top 5 solid tumor-related concepts significantly associated with PES. Table S4. Gene expression TaqMan assays. All assays were purchased from Life Technologies and were pre-designed for the probe to span exons except for snoRNA55, for which both the primers and probe map within a single exon. All probes had the FAM reporter signal.

Additional file 2: Figure S1. Expression of PCGEM1 in LTL-331 castration time series. A, Expression ( $(P C R)$ of the labeled genes in LTL-331 tumor line from mice supplemented with testosterone (Test.) $(n=3,5.0 \mathrm{mg} / \mathrm{mouse})$, or after surgical castration $(n=1$, at $1,2,3,8$ and 12 weeks). Expression of all genes is referenced to average expression levels of HPRT1 and GAPDH and is expressed relative to the gene's expression in testosterone-supplemented mice. Dots represent mean expression value and bars represent standard deviation. B, Serum PSA levels from the corresponding LTL-331 xenograft bearing animals in A. Figure S2. PCGEM1 basal expression profile in a panel of PCa cell lines. Expression levels of PCGEM1 in a non-neoplastic prostate cell line and a panel of commonly used AR+ and AR- PCa cell line models. PCGEM1 expression is referenced to average expression levels of HPRT1 and GAPDH and is expressed relative to its expression in 22RV1 cells where it is barely expressed (Ct value $=\sim 41$ ). No column represents that PCGEM1 transcript was undetectable in the corresponding cell line using the Ct cutoff of 45 cycles. Columns represent mean expression value and bars represent standard deviation. Figure S3. Sub-cellular localization of PCGEM1 with or without DHT treatment in vitro. Expression of the labelled genes (qPCR) in sub-cellular fractions of $A$, untreated LNCaP cells and B, LNCaP cells stimulated with DHT (10nM) for $12 \mathrm{~h}$. Columns represent mean distribution value from 2 independent experiments and bars represent standard deviation. Figure S4. Taylor PCa cohort analysis. PCGEM1 expression (microarray data) in 131 primary PCa, 19 partially paired secondary metastatic PCa tissues and 29 normal prostatic tissues. These are median-centered values where bars represent maximum and minimum value per group. NS, not significant; *** $p 0.001$ (2-Way ANOVA and Tukey's post-test).

Additional file 3: Supplementary methods and materials.

\section{Competing interests}

All authors declare that they have no competing interests.

\section{Authors' contributions}

AP and FC conceived and designed the study with guidance from YZW and CDH. AP and FC performed the CBioPortal and Oncomine analysis. AP performed the in vitro experiments, RNA extractions and gene quantifications, analyzed and interpreted data, and wrote the entire manuscript and prepared the figures. HX and YW did the in vivo experiments. FM, VRR and CC performed genomic analysis. HHL, DL, NRNS and HC helped in sample preparation. PLC critically revised the paper. YZW and $\mathrm{CDH}$ participated in designing experiments and critically revised the paper as well. In addition, all authors read and approved the final manuscript.

\section{Acknowledgements}

This work was financially supported by Canadian Cancer Society Research Institute, grant number: $701097(\mathrm{CDH})$; Canadian Institutes of Health Research, grant numbers: 102604-1, 119991-1, 123449-1 (YZW); Prostate Cancer Canada (YZW); Terry Fox Research Institute, grant number: 116129-1 (YZW); Michael Smith Foundation for Health Research Fellowship, number: 5629 (FC); and BC Prostate Cancer Foundation "grant-in-aid" award (FC).

\section{Author details}

${ }^{1}$ Honours Biotechnology Program, Department of Microbiology and Immunology, University of British Columbia, Vancouver, BC, Canada. ${ }^{2}$ Experimental Therapeutics, BC Cancer Research Centre, 675 West 10th Avenue, Vancouver, BC V5Z 1L3, Canada. ${ }^{3}$ The Vancouver Prostate Centre, Vancouver General Hospital, Vancouver, BC, Canada. Interdisciplinary Oncology Program, Faculty of Medicine, University of British Columbia, Vancouver, BC, Canada. ${ }^{5}$ Department of Urologic Sciences, University of British Columbia, Vancouver, BC, Canada. ${ }^{6}$ Department of Surgery, University of British Columbia, Vancouver, BC, Canada.

Received: 18 November 2014 Accepted: 5 February 2015

Published online: 21 February 2015

\section{References}

1. Wapinski O, Chang HY. Long noncoding RNAs and human disease. Trends Cell Biol. 2011;21(6):354-61.

2. Walsh AL, Tuzova AV, Bolton EM, Lynch TH, Perry AS. Long noncoding RNAs and prostate carcinogenesis: the missing 'linc'? Trends Mol Med. 2014;20(8):428-36.

3. Derrien T, Johnson R, Bussotti G, Tanzer A, Djebali S, Tilgner H, et al. The GENCODE v7 catalog of human long noncoding RNAs: analysis of their gene structure, evolution, and expression. Genome Res. 2012;22(9):1775-89.

4. Crea F, Clermont PL, Parolia A, Wang Y, Helgason CD. The non-coding transcriptome as a dynamic regulator of cancer metastasis. Cancer Metastasis Rev. 2013;33:1-16.

5. Prensner JR, Chinnaiyan AM. The emergence of IncRNAs in cancer biology. Cancer Discov. 2011;1(5):391-407.

6. Prensner JR, lyer MK, Balbin OA, Dhanasekaran SM, Cao Q, Brenner JC, et al. Transcriptome sequencing across a prostate cancer cohort identifies PCAT-1, an unannotated lincRNA implicated in disease progression. Nat Biotechnol. 2011;29(8):742-9.

7. Prensner JR, lyer MK, Sahu A, Asangani IA, Cao Q, Patel L, et al. The long noncoding RNA SChLAP1 promotes aggressive prostate cancer and antagonizes the SWI/SNF complex. Nat Genet. 2013;45:1392-8.

8. Bussemakers MJ, van Bokhoven A, Verhaegh GW, Smit FP, Karthaus HF, Schalken JA, et al. DD3: a new prostate-specific gene, highly over expressed in prostate cancer. Cancer Res. 1999:59(23):5975-9.

9. Srikantan V, Zou Z, Petrovics G, Xu L, Augustus M, Davis L, et al. PCGEM1, a prostate-specific gene, is overexpressed in prostate cancer. Proc Natl Acad Sci U S A. 2000;97(22):12216-21.

10. Chung S, Nakagawa H, Uemura M, Piao L, Ashikawa K, Hosono N, et al. Association of a novel long non-coding RNA in 8q24 with prostate cancer susceptibility. Cancer Sci. 2011;102(1):245-52.

11. Mehra R, Shi Y, Udager AM, Prensner JR, Sahu A, lyer MK, et al. A novel RNA in situ hybridization assay for the long noncoding RNA SChLAP1 predicts poor clinical outcome after radical prostatectomy in clinically localized prostate cancer. Neoplasia. 2014;16(12):1121-7. 
12. Roobol MJ, Schroder FH, van Leeuwen $P$, Wolters $T$, van den Bergh RC, van Leenders GJ, et al. Performance of the prostate cancer antigen 3 (PCA3) gene and prostate-specific antigen in prescreened men: exploring the value of PCA3 for a first-line diagnostic test. Eur Urol. 2010;58:475-81.

13. Crea F, Watahiki A, Quagliata L, Xue H, Pikor L, Parolia A, et al. Identification of a long non-coding RNA as a novel biomarker and potential therapeutic target for metastatic prostate cancer. Oncotarget. 2014;5(3):764-74.

14. Fu X, Ravindranath L, Tran N, Petrovics G, Srivastava S. Regulation of apoptosis by a prostate-specific and prostate cancer-associated noncoding gene, PCGEM1. DNA Cell Biol. 2006;25(3):135-41.

15. Petrovics G, Zhang W, Makarem M, Street JP, Connelly R, Sun L, et al. Elevated expression of PCGEM1, a prostate-specific gene with cell growth-promoting function, is associated with high-risk prostate cancer patients. Oncogene. 2004;23(2):605-11

16. Yang L, Lin C, Jin C, Yang JC, Tanasa B, Li W, et al. LncRNA-dependent mechanisms of androgen-receptor-regulated gene activation programs. Nature. 2013:500(7464):598-602.

17. Prensner JR, Sahu A, lyer MK, Malik R, Chandler B, Asangani IA, et al. The IncRNAs PCGEM1 and PRNCR1 are not implicated in castration resistant prostate cancer. Oncotarget. 2014;5(6):1434-8.

18. Balk SP, Knudsen KE. AR, the cell cycle, and prostate cancer. Nucl Recept Signal. 2008;6:e001.

19. Sharifi N, Gulley JL, Dahut WL. Androgen deprivation therapy for prostate cancer. JAMA. 2005;294(2):238-44.

20. Mills IG. Maintaining and reprogramming genomic androgen receptor activity in prostate cancer. Nat Rev Cancer. 2014;14(3):187-98.

21. Fry CJ, Farnham PJ. Context-dependent transcriptional regulation. J Biol Chem. 1999:274(42):29583-6.

22. Sharma NL, Massie CE, Ramos-Montoya A, Zecchini V, Scott HE, Lamb AD, et al. The androgen receptor induces a distinct transcriptional program in castration-resistant prostate cancer in man. Cancer Cell. 2013;23(1):35-47.

23. Lin D, Wyatt AW, Xue H, Wang Y, Dong X, Haegert A, et al. High fidelity patient-derived xenografts for accelerating prostate cancer discovery and drug development. Cancer Res. 2014;74(4):1272-83.

24. DePrimo SE, Diehn M, Nelson JB, Reiter RE, Matese J, Fero M, et al. Transcriptional programs activated by exposure of human prostate cancer cells to androgen. Genome Biol. 2002;3(7):RESEARCH0032

25. Luo M, Li Z, Wang W, Zeng Y, Liu Z, Qiu J. Long non-coding RNA H19 increases bladder cancer metastasis by associating with $\mathrm{EZH} 2$ and inhibiting E-cadherin expression. Cancer Lett. 2013;333(2):213-21.

26. Cai $X$, Cullen BR. The imprinted $H 19$ noncoding RNA is a primary microRNA precursor. RNA. 2007;13(3):313-6.

27. Tsang WP, Ng EK, Ng SS, Jin H, Yu J, Sung JJ, et al. Oncofetal H19-derived miR-675 regulates tumor suppressor RB in human colorectal cancer. Carcinogenesis. 2010;31(3):350-8.

28. Taylor BS, Schultz N, Hieronymus H, Gopalan A, Xiao Y, Carver BS, et al. Integrative genomic profiling of human prostate cancer. Cancer Cell. 2010;18:11-22.

29. Xue Y, Wang M, Kang M, Wang Q, Wu B, Chu H, et al. Association between Incrna PCGEM1 polymorphisms and prostate cancer risk. Prostate Cancer Prostatic Dis. 2013;16(2):139-44.

\section{Submit your next manuscript to BioMed Central and take full advantage of:}

- Convenient online submission

- Thorough peer review

- No space constraints or color figure charges

- Immediate publication on acceptance

- Inclusion in PubMed, CAS, Scopus and Google Scholar

- Research which is freely available for redistribution 